\title{
Multicast Routing with End-to-End Delay and Delay Variation Constraints
}

\author{
George N. Rouskas Ilia Baldine
}

TR-95-09

July 1995

\begin{abstract}
We study the problem of constructing multicast trees to meet the quality of service requirements of real-time, interactive applications operating in high-speed packet-switched environments. In particular, we assume that multicast communication depends on (a) bounded delay along the paths from the source to each destination, and (b) bounded variation among the delays along these paths. We first establish that the problem of determining such a constrained tree is $\mathcal{N} \mathcal{P}$-complete. We then derive heuristics that demonstrate good average case behavior in terms of the maximum inter-destination delay variation of the final tree. In addition, our heuristics achieve their best performance under conditions typical of multicast scenarios in high-speed networks. We also show that it is possible to dynamically reorganize the initial tree in response to changes in the destination set, in a way that is minimally disruptive to the multicast session.
\end{abstract}

Department of Computer Science

North Carolina State University

Raleigh, NC 27695-8206 


\section{Introduction}

In multicast communication messages are concurrently sent to multiple destinations, all members of the same multicast group. Mechanisms to support such a form of communication are becoming an increasingly important component of the design and implementation of distributed systems [1]. One of the core issues that needs to be addressed as part of providing such mechanisms is the issue of routing, which primarily refers to the determination of a set of paths to be used for carrying the messages from the source to the destinations nodes. For reasons related to the efficient use of network resources involved in a multicast session, typical approaches to multicast routing require the transmission of packets along the branches of a tree spanning the source and destination nodes.

The problem of computing multicast trees has received considerable attention in the past, and several algorithms have been proposed based on a number of optimization goals. One frequently considered optimization objective is to minimize the total cost of the tree, which is taken as the sum of the costs on the links of the multicast tree. The minimum cost tree is known as the Steiner tree [2], and finding such a tree is a well-known $\mathcal{N} \mathcal{P}$-complete problem [3]. Heuristics to construct trees of low overall cost have been developed in $[4,5,6,7]$.

While total tree cost as a measure of bandwidth efficiency is certainly an important parameter, it is not sufficient to characterize the quality of the tree as perceived by the interactive multimedia and real-time applications which are expected to utilize emerging high-speed networks. More specifically, networks supporting real-time traffic will be required to provide certain quality of service guarantees in terms of the end-to-end delay along the individual paths from the source to each of the destination nodes. The problem of routing multicast traffic with real-time constraints has been studied in $[8,9]$ and heuristics to compute low-cost trees which guarantee an upper bound on the end-to-end delay have been developed. For a survey and extensive simulation study of a large number of existing multicast algorithms, and an evaluation of their performance in high-speed real-time environments, the reader is referred to $[10,11]$.

In this work we consider an additional criterion that can be used to characterize the quality of the multicast tree for interactive, real-time applications. In particular, we assume that, in addition to end-to-end delay bounds, the multicast tree must also guarantee bounds on the variation among the delays along the individual source-destination paths. One can think of such a bound as providing synchronization among the various receivers, in order to ensure that no receiver is "left behind" and none is "far ahead" during the lifetime of the session. Although delay variation has not, to the best of our knowledge, been considered in the design of multicast tree algorithms, the maximum delay variation among the paths of the final tree was one of the performance metrics included in 
the comparative study in $[10,11]$.

There are several situations in which the need for bounded variation among the end-to-end delays arises. During a teleconference, for instance, it is important that the current speaker be heard by all participants at the same time, or else the communication may lack the feeling of an interactive face-to-face discussion. As another example, consider the use of multicast messages to update multiple copies of a replicated data item (or file) in a distributed database system. Minimizing the delay variation in this case would minimize the length of time during which the database is in an inconsistent state. Furthermore, being able to look at the information carried by the multicast message long before others can do the same, might, for certain applications, translate into gaining a competitive edge. A distributed game scenario in which a number of players are connected over the network to a game server, and compete against each other using information sent by the server to their screens, would be one such example.

Following this introduction, Section 2 presents a model that captures the salient features of multicast communication over packet-switched networks. In Section 3 we show that the problem of constructing trees to guarantee a bound on the variation of the end-to-end delays along the sourcedestination paths is $\mathcal{N} \mathcal{P}$-complete. In Section 4 we develop heuristic algorithms for this problem; we also outline an approach to dynamically reorganizing the initial tree as nodes are added to, or deleted from the multicast group. We present some numerical results in Section 5, and conclude the paper in Section 6.

\section{Network Model for Multicasting}

We consider the routing of multicast connections in a packet-switched communication network. The network is represented by a weighted directed graph $G=(V, A)$, where $V$ denotes the set of nodes, and $A$, the set of arcs, corresponds to the set of communication links connecting the various nodes. We will use $n=|V|$ to refer to the number of nodes in the network. Without loss of generality, we only consider graphs with at most one arc between an ordered pair of nodes.

We define a link-delay function $\mathcal{D}: A \rightarrow \mathcal{R}^{+}$which assigns a non-negative weight to each link in the network. More specifically, the value $\mathcal{D}(\ell)$ associated with link $\ell \in A$ is a measure of the total delay that data packets experience on that link, including the queueing, transmission, and propagation components. Suppose now that both links $\ell=(v, u)$ and $\ell^{\prime}=(u, v)$ exist for some $v, u \in V$. Since, in practice, communication networks can be asymmetric in nature, we allow link-delay functions that assign different values to links $\ell$ and $\ell^{\prime}$, i.e., it is possible that $\mathcal{D}(\ell) \neq \mathcal{D}\left(\ell^{\prime}\right)$. 
Under the multicast routing scenario we are considering, packets originating at some source node $s \in V$ in the network have to be delivered to a number of destinations. We will call this set $M \subseteq V-\{s\}$ of destination nodes the destination set or multicast group, and will use $m=|M|$ to denote its size. In general, several multicast sessions may proceed concurrently within the network, each characterized by a source node and a destination set.

We assume that communication in the network is connection-oriented, and that multicast connections are established by issuing a connect request; similarly, at the conclusion of a session a disconnect request is issued. In response to a connect request, and prior to any data been transferred from the source to the destinations, a connection establishment process is initiated. Central to the connection establishment is the determination of routes between the source and the destinations, over which data packets and acknowledgements will be carried for the duration of the multicast session.

Let $s$ and $M$ be the source and multicast group, respectively, of a certain multicast session. We assume that multicast packets for this session are routed from $s$ to the destinations in $M$ via the links of a multicast tree $T=\left(V_{T}, A_{T}\right)$ rooted at $s$. This tree is constructed during the route determination phase of the connection establishment process, based on information supplied as part of the connect request (more on this shortly). The multicast tree is a subgraph of $G$ (i.e., $V_{T} \subseteq V$ and $A_{T} \subseteq A$ ) spanning $s$ and the nodes in $M$ (that is, $M \cup\{s\} \subseteq V_{T}$ ). In addition, $V_{T}$ may contain relay nodes, that is, nodes intermediate to the path from the source to a destination. Relay nodes are not consumers of multicast packets; rather, they simply forward these packets along the downstream links of the multicast tree, and also forward acknowledgements from the destination nodes towards the source, along the upstream links.

Let $T$ be a multicast tree for the source-multicast group pair $(s, M)$, and let $P_{T}(s, v)$ denote the unique path from source $s$ to destination $v \in M$ in the tree $T$. Then, multicast packets from $s$ to $v$ experience a total delay of $\sum_{\ell \in P_{T}(s, v)} \mathcal{D}(\ell)$ along this path. We now introduce two parameters that can be used to characterize the quality of the multicast tree as perceived by the application performing the multicast. These parameters relate end-to-end delays along individual source-destination paths to the desired level of quality of service, as follows.

- Source-destination delay tolerance, $\Delta$. Parameter $\Delta$ represents an upper bound on the acceptable end-to-end delay along any path from the source to a destination node. This parameter reflects the fact that the information carried by multicast packets becomes stale $\Delta$ time units after its transmission at the source, and as such, it is of no value to the receivers.

- Inter-destination delay variation tolerance, $\delta$. Parameter $\delta$ is the maximum difference between 
the end-to-end delays along the paths from the source to any two destination nodes that can be tolerated by the application. In essence, this parameter defines a synchronization window for the various receivers.

By supplying values for parameters $\Delta$ and $\delta$, the application in effect imposes a set of constraints on the paths of the multicast tree. The application will proceed only if a tree satisfying these constraints can be found; otherwise, the application will abort. In the following section we take a closer look at the problem of determining multicast trees that guarantee a desired level of performance in terms of the quality of service criteria discussed above.

\section{Delay- and Delay Variation-Bounded Multicast Trees}

Let $\Delta$ and $\delta$ be the delay and delay variation tolerances, respectively, as specified by a higher level application that wishes to initiate a multicast session. Our objective is to determine a multicast tree such that delays along all source-destination paths in the tree are within the two tolerances. This problem, which we will call the Delay-and Delay Variation-Bounded Multicast Tree (DVBMT) problem, arises naturally as a decision problem, and can be formally expressed as follows.

Problem 3.1 (DVBMT) Given a network $G=(V, A)$, a source node $s \in V$, a multicast group $M \subseteq V-\{s\}$, a link-delay function $\mathcal{D}: A \rightarrow \mathcal{R}^{+}$, a delay tolerance $\Delta$, and a delay variation tolerance $\delta$, does there exist a tree $T=\left(V_{T}, A_{T}\right)$ spanning $s$ and the nodes in $M$, such that:

$$
\begin{gathered}
\sum_{\ell \in P_{T}(s, v)} \mathcal{D}(\ell) \leq \Delta \quad \forall v \in M \\
\left|\sum_{\ell \in P_{T}(s, v)} \mathcal{D}(\ell)-\sum_{\ell \in P_{T}(s, u)} \mathcal{D}(\ell)\right| \leq \delta \quad \forall v, u \in M
\end{gathered}
$$

We will refer to (1) as the source-destination delay constraint, while (2) will be called the interdestination delay variation constraint. We will also say that tree $T$ is a feasible tree for a multicast session with source $s$ and destination set $M$, if and only if $T$ satisfies both (1) and (2). Note that, in order for the multicast session to proceed, it is necessary and sufficient that a single feasible tree be constructed, as any feasible tree can meet the quality of service requirements as expressed by parameters $\Delta$ and $\delta$.

An interesting observation regarding constraints (1) and (2) is that they represent two conflicting objectives. Indeed, the delay constraint (1) dictates that short paths be used. But choosing the shortest paths may lead to a violation of the delay variation constraint among nodes that are close 
to the source and nodes that are far away from it. Consequently, it may be necessary to select longer paths for some nodes in order to satisfy the latter constraint. Then, the problem of finding a feasible tree for $D V B M T$ becomes one of selecting paths in a way that strikes a balance between these two objectives.

The source-destination constraint (1) has been previously considered in the context of designing constrained Steiner trees for real-time, interactive applications [8, 9]. To the best of our knowledge, however, our work is the first to explicitly consider the inter-destination delay variation constraint (2) in the construction of multicast trees. This should not, however, be taken to mean that the importance of providing guarantees on the maximum value of the end-to-end delay variation has not been recognized. In fact, as part of a recent study $[10,11]$ to evaluate the relative performance of a large number of multicast algorithms and their suitability to high-speed real-time applications, the following quantity was measured and used as a criterion in the evaluation:

$$
\delta_{T}=\max _{u, v \in M}\left\{\left|\sum_{\ell \in P_{T}(s, u)} \mathcal{D}(\ell)-\sum_{\ell \in P_{T}(s, v)} \mathcal{D}(\ell)\right|\right\}
$$

Quantity $\delta_{T}$ is the maximum inter-destination delay variation in tree $T$, and, given a value for $\delta$, it can be used to determine whether tree $T$ can meet the quality of service requirements of the application. According to the study, none of the existing algorithms provides good performance in terms of $\delta_{T}$; this is not surprising, as none of the algorithms considered in $[10,11]$ takes the delay variation constraint (2) into account. Our work addresses the problem of designing multicast algorithms that overcome this inefficiency.

Before proceeding, we would like to resolve the open question regarding the existence of efficient algorithms for DVBMT. Unfortunately, the following theorem establishes that DVBMT is $\mathcal{N} \mathcal{P}$-complete, implying that a polynomial-time algorithm that determines a feasible tree for any arbitrary instance of this problem is unlikely to be found.

Theorem 3.1 DVBMT is $\mathcal{N} \mathcal{P}$-complete whenever the size of the multicast group $|M| \geq 2$.

Proof. See Appendix A.

The next section introduces a heuristic approach to determining feasible trees for any arbitrary instance of $D V B M T$. 


\section{Multicast Tree Algorithms for DVBMT}

Consider an application running at node $s$, and suppose that the application issues a request for establishing a multicast connection with destination set $M$. Along with the request, the application also supplies values for the path delay tolerance $\Delta$, and inter-destination delay variation tolerance $\delta$; these values reflect certain quality of service requirements which the network must guarantee in order for the multicast session to proceed. As part of the connection establishment process, upon receiving the request, a multicast tree satisfying constraints (1) and (2) needs to be determined. In this section we present algorithms that can be used to construct such a tree. Our algorithms operate under the assumption that complete information regarding the network topology is stored locally at node $s$, making it possible to determine the multicast tree at the source itself. This information may be collected and updated using one of several existing topology-broadcast algorithms [12].

The sequence of actions taken by node $s$ during the course of constructing a multicast tree is illustrated in the flowchart of Figure 1, where we have assumed that the values of the delay and delay variation tolerances $\Delta$ and $\delta$, respectively, provided by the application are negotiable ${ }^{1}$. As a first step, the tree of shortest paths from $s$ to all nodes in $M$ is constructed; this can be achieved using Dijkstra's algorithm [13], which, for dense graphs with $n$ nodes, takes time $\mathcal{O}\left(n^{2}\right)$. Let $T_{0}$ be this tree of shortest paths. If $T_{0}$ does not satisfy the path delay constraint (1) no tree may satisfy it, implying that the delay tolerance $\Delta$ supplied by the application is too tight; negotiation may then be necessary to determine a looser value of $\Delta$. Suppose now that the (original or negotiated) value of $\Delta$ is such that the delay requirement (1) is met for tree $T_{0}$. If $T_{0}$ also meets the delay variation requirement (2) then $T_{0}$ is a feasible tree for this instance of the $D V B M T$ problem, and the multicast session may take place over the tree of shortest paths. As a result, the route determination phase completes successfully, and the connection establishment process may then proceed to a subsequent phase (such as bandwidth reservation along the paths of the tree, etc.).

On the other hand, it is possible that tree $T_{0}$ fail to satisfy constraint (2). In that case, our approach is to have the source execute a search algorithm in an attempt to construct a new tree in which the delays along all source-destination paths satisfy both (1) and (2). Based on the results of the previous section, however, an algorithm that efficiently solves any arbitrary instance of $D V B M T$

\footnotetext{
${ }^{1}$ Using values for parameters $\Delta$ and $\delta$ other than the ones recommended by the application may result in a degradation of the quality of service as perceived by some or all of the receivers in the multicast group. However, as long as the negotiated values do not differ significantly from the original ones (in which case a basic grade of service can be guaranteed), it may be possible for the multicast session to proceed. It is also conceivable that incentives to join the multicast session be offered to receivers expected to experience a lower quality of service as a result of the network's inability to guarantee the initial requirements.
} 


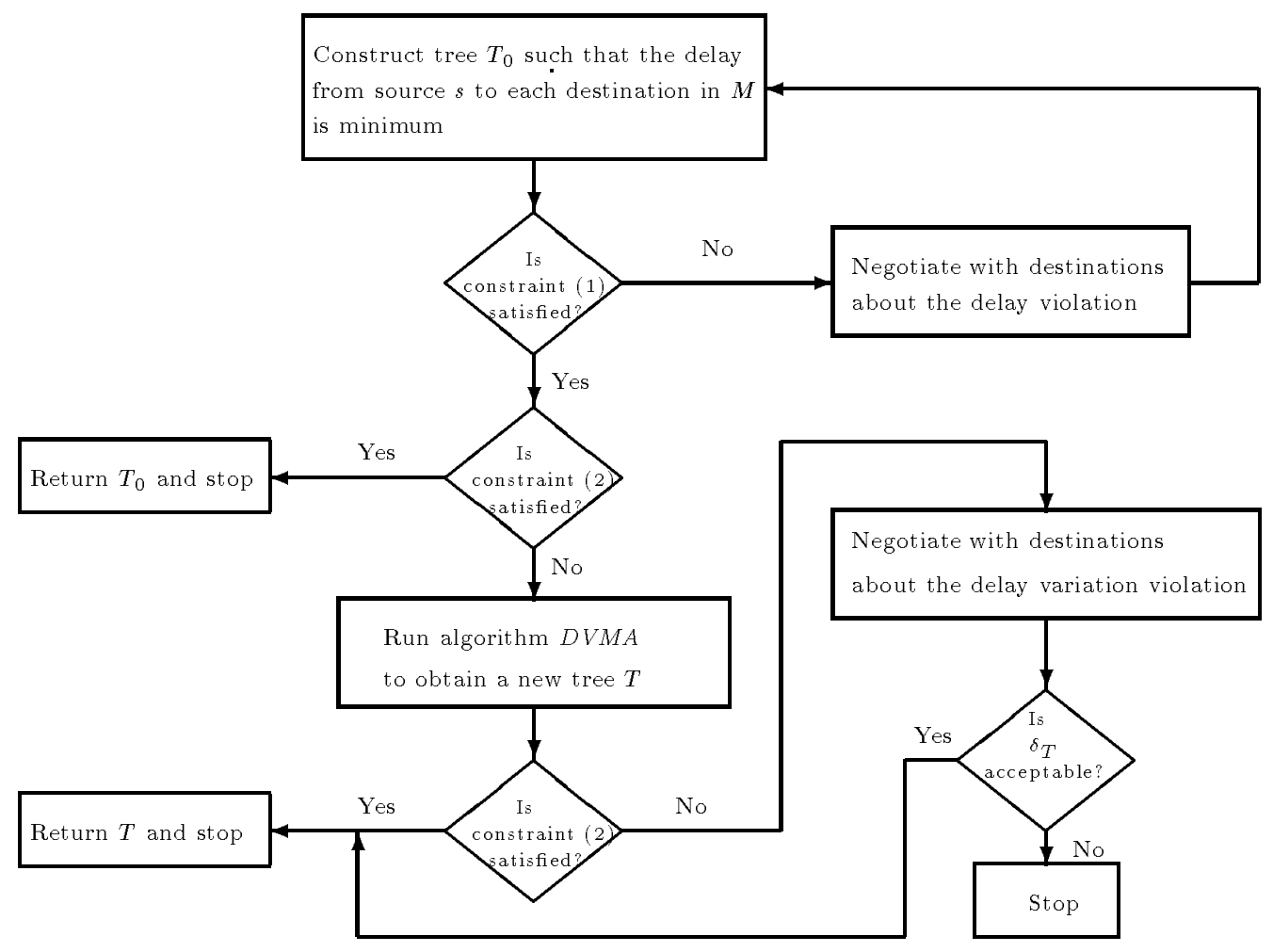

Figure 1: Flowchart of our approach to obtaining a multicast tree for the DVBMT problem

may not exist, meaning that the search algorithm has to employ a heuristic approach. Nevertheless, suppose that a heuristic algorithm is available, and that it returns a tree which constitutes a solution to the given instance of the $D V B M T$ problem; then a tree for the multicast session has been found.

However, a heuristic algorithm may fail to discover a feasible tree, either because no such tree exists or because of the ineffectiveness of the search strategy employed. Other than abandoning the connection altogether, the only course of action available to the application at that point would be to initiate another round of negotiations in hope of determining a new value for the delay tolerance $\delta$, one that would be acceptable to all parties involved in the multicast session. If such a value can be agreed upon the source would go through another iteration in the flowchart of Figure 1, otherwise the multicast session would have to be abandoned.

An alternative that would result in a considerable speed-up of the negotiation process would be to design the search algorithm so that it always returns, among the trees considered, the one with the smallest value of $\delta_{T}$ in $(3)$. Indeed, regardless of whether a solution to the given instance of DVBMT problem exists or not, the tree corresponding to the smallest value of $\delta_{T}$ is the best tree 
that can be obtained with the search algorithm at hand. If this tree is available at the termination of the algorithm, all that has to be determined during the negotiation process is whether an acceptable level of quality of service can be sustained for the given value of $\delta_{T}$ and there is no need to repeat the route determination process; this is shown in Figure 1.

The following subsection presents DVMA, a new multicast tree heuristic designed to solve the $D V B M T$ problem. Following that, we show how the basic idea behind $D V M A$ can be used to develop a solution to the corresponding dynamic problem, i.e., the problem of updating the multicast tree in response to receiver requests for joining or leaving an ongoing multicast session.

\subsection{Delay Variation Multicast Algorithm (DVMA)}

Let $T_{0}$ be the tree of shortest paths from source $s$ to the nodes in the destination set $M$ for the multicast connection under consideration. Let us also assume that $T_{0}$ meets the delay requirement (1), but that it does not meet the delay variation requirement (2). The Delay Variation Multicast Algorithm (DVMA), described in detail in Figure 2, can then be used to search through the space of candidate trees (i.e., trees spanning $s$ and the nodes in $M$ ) for a feasible solution to the DVBMT problem. DVMA either returns a feasible tree, or, having failed to discover such a tree, it returns one which (a) satisfies the delay constraint (1) and (b) has the least value of $\delta_{T}$ among the trees considered by the algorithm. The basic idea behind the operation of DVMA is now described.

Let $M$ be the destination set, and assume for the moment that a feasible tree $T=\left(V_{T}, A_{T}\right)$ spanning $s$ and a subset of $M$ has already been determined. Let $U=M-\left(M \cap V_{T}\right)$ be the set of destination nodes not in the tree $T$; in other words, no paths from the source $s$ to the nodes in $U$ have been determined yet. DVMA operates by appropriately augmenting tree $T$ to eventually include all nodes in $U$; to this end, it repeats the following three steps as long as $U \neq \phi$ :

1. Select a destination node $u \in U$.

2. Find a "good" path from a node $v \in V_{T}$ to $u$ that uses no nodes in $V_{T}$ other than $v$, and no links in $A_{T}$; note that $v$ could be the source node $s$.

3. Construct a new tree $T^{\prime}$ by including all nodes and links of this path to the initial tree $T$, and update $U$ to exclude $u$ and any other destination nodes along this path.

The second step is crucial to the operation of DVMA, and warrants further explanation. Recall that our objective is to construct a feasible tree that includes all nodes in $M$, therefore a "good" path in Step 2 above is one which, if connected to $T$ in Step 3, the resulting tree $T^{\prime}$ would be a 
feasible tree for the subset of the set of destination nodes it contains. In order to find such a path, we construct the $l$ shortest paths from a node $v$ of $T$ to $u$. The graph used to find these paths is created by excluding all nodes of $T$ other than $v$, and all links of $T$ from the original graph $G$. The exclusion of these nodes and links from $G$ guarantees that connecting any of the $l$ paths so constructed to $T$ will not create a cycle.

It is possible, though, that none of the $l$ paths from $v$ to $u$ will yield a feasible tree. For this reason, we repeat the process for all nodes $v \in V_{T}$ in an attempt to find a "good" path between any $v \in V_{T}$ and $u$. Even so, the algorithm may still not be able to find such a path; for instance, a feasible tree for this destination set may not exist in the first place. Recall, however, that we would like the algorithm to return the best tree (in terms of maximum inter-destination delay variation) it can find. We now modify our definition of a "good" path so that, if a path yielding a feasible tree $T^{\prime}$ can not be found, a "good" path is one which

(a) the total delay from $s$ to $u$ (i.e., the delay from $s$ to $v$ in $T$, plus the delay from $v$ to $u$ over the path) is at most $\Delta$, and

(b) the tree $T^{\prime}$ created by connecting this path to $T$ has the least value of maximum delay variation among the trees constructed by connecting the other paths to $T$.

In essence, the purpose of the greedy rule (b) above is to prune the search space, i.e., to prevent certain candidate trees from receiving further consideration.

The only question that remains to be answered then, is how an initial tree $T$ is constructed. To answer this question consider $T_{0}$, the tree of shortest paths, which, by hypothesis, does not satisfy the delay variation constraint (2). Let $w$ be the destination node with the longest path in this tree. Since it is not possible to make the delay from $s$ to $w$ any smaller than the delay incurred over the path from $s$ to $w$ in $T_{0}$, the only alternative to constructing a feasible tree is to find longer paths from $s$ to some or all of the other destination nodes. Hence, our approach is to start with an initial tree $T$ consisting only of the shortest path from $s$ to $w$, and repeat the three steps described above to create a feasible tree that will include all other destination nodes.

To complete the description of the search strategy employed by DVMA, note that it is possible that no feasible tree for the given destination set includes the shortest path from $s$ to $w$. However, if a feasible tree exists, it will contain some path from $s$ to $w$. Therefore, if the process of constructing a feasible tree starting from the shortest path from $s$ to $w$ fails, the second shortest path from $s$ to $w$ is considered as the initial tree and the process is repeated. Our search for a feasible tree terminates when one is found, or when trees based on the first $k$ shortest paths from $s$ to $w$ have 
been constructed, whichever occurs first. In the latter case, the algorithm will return the tree with the smallest value of $\delta_{T}$ in (3). The details of the resulting algorithm ( $\left.D V M A\right)$ can be found in Figure 2.

The correctness of DVMA is provided by the following lemma. Note, however, that although the algorithm returns the best tree, in terms of maximum delay variation, that it can find, because of its heuristic nature it may fail to discover a feasible tree for the given value of $\delta$ even if one exists.

Lemma 4.1 (Correctness of DVMA) Algorithm DVMA returns a tree $T$ spanning $s$ and all nodes $v \in M$. The tree $T$ satisfies constraint (1), and either satisfies constraint (2), or is the one with the smallest value of $\delta_{T}$ in (3) among the trees considered by the algorithm.

Proof. We will first show that the algorithm returns a tree $T$ spanning $s$ and the nodes in $M$. If DVMA returns $T_{0}$, there is nothing to prove. Otherwise, $T$ is one of the $T_{i}$ 's constructed during one iteration of the loop that starts at line 4. $T$ is initialized to some path $p_{i}$ at line 5; clearly, at this point $T$ is a tree containing the source $s$ and at least one more destination $w \in M$. New nodes and links are added to $T$ in line 15 , where a new path $q$ from a node in $v \in V_{T}$ to a node $u \in M, u \notin V_{T}$ is incorporated. The resulting new graph is a tree as path $q$ cannot contain any nodes or links of $T$ other than $v$ itself. Indeed, all other nodes and links of $T$ were removed at line 10 , before path $q$ was determined. The new tree $T$ has at least one more node, $u \in M$; since $s$ was in the tree initially, no nodes are ever removed from $T$, and paths are added to it until all destinations in $M$ are in $T$, our first claim is true.

That the delay constraint (1) is satisfied by the final tree $T$ is now is easy to see. If $T=T_{0}$ this is true by hypothesis; if $T \neq T_{0}$ this is also true as no path is ever added to any tree $T_{i}$ unless the delay constraint is satisfied (refer to lines 3 and 12). Finally, if the algorithm terminates at line 18, the tree returned is a feasible one; otherwise, line 19 guarantees that the tree returned is the one with the smallest value of $\delta_{T}$ among the ones constructed during the execution of the algorithm. $\square$

The next lemma determines the running time complexity of $D V M A$.

Lemma 4.2 The worst-case complexity of DVMA is $\mathcal{O}\left(k l m n^{4}\right)$, where $k$ is the number of paths generated at line 3 of Figure $2, l$ the number of paths generated at line 11, $m=|M|$ is the number of destinations in the multicast group $M$, and $n=|V|$ is the number of nodes in the network.

Proof. The running time of DVMA is dominated by the iteration between lines 4 and 20; this outer loop is executed at most $k$ times. During one iteration of the outer loop, the "while" loop at line 7 is executed at most $m-1$ times. Let $t_{j}$ be the number of nodes in the tree during the $j$-th 


\section{Delay Variation Multicast Algorithm (DVMA)}

The algorithm is executed if $T_{0}$, the tree of shortest paths, satisfies constraint (1) but does not satisfy constraint (2). We let $w \in M$ be a node such that $\sum_{\ell \in P_{T_{0}}(s, w)} \mathcal{D}(\ell)=\max _{v \in M}\left\{\sum_{\ell \in P_{T_{0}}(s, v)} \mathcal{D}(\ell)\right\}$.

1. begin

2. Let $T=T_{0} \quad / / T$ is the tree returned by the algorithm

3. Find the first $k$ shortest paths from $s$ to $w$ in the original graph $G=(V, A)$, such that the delay from $s$ to $w$ over these paths is less than $\Delta$; label these paths $p_{1}, \ldots, p_{k}$ in increasing order of delay

4. for $i=1$ to $k$ do $\quad / /$ construct a multicast tree $T_{i}$ for each path $p_{i}$

5. Initialize $T_{i}=\left(V_{i}, A_{i}\right)$ to include all the nodes and links of path $p_{i}$; obviously, $s, w \in V_{i}$

6. Let $U=M-\left(M \cap V_{i}\right)$ be the set of destinations not yet connected to the tree $T_{i}$

7. $\quad$ while $U \neq \phi$ do

8. Pick any node $u \in U \quad / /$ will connect $u$ to the tree $T_{i}$

9. for each node $v \in V_{i}$ do $\quad / /$ find a path from $v$ to $u$

10. Construct a new graph $G^{\prime}$ starting with the initial graph $G$ and excluding all nodes in $V_{i}-\{v\}$ and all links in $A_{i}$

11. Find the first $l$ shortest paths from $v$ to $u$ in the new graph $G^{\prime}$

12. Of these $l$ paths choose the best one (as described in Section 4.1) and call it $q_{v}$

13. $\quad$ end of for each node $v \in V_{i}$ loop

14. Select the best path $q$ among all paths $q_{v}, v \in V_{i}$ (as in Step 12 above)

15. Update $T_{i}=\left(V_{i}, A_{i}\right)$ to include all nodes and links in path $q$

16. Update $U=M-\left(M \cap V_{i}\right)$

$/ /$ node $u$, and possibly other nodes in $U$ have now been connected to $T_{i}$

17. end of while loop // construction of tree $T_{i}$ has been completed

18. If tree $T_{i}$ satisfies constraint (2) return $T_{i}$ and stop

19. Let $T$ be the tree among $T$ and $T_{i}$ with the smallest value of $\delta_{T}$ in (3)

20. end of for $i$ loop

21. return $T$ // no tree satisfied the inter-destination delay variation constraint

22. end of the algorithm

Figure 2: Heuristic algorithm for the DVBMT problem 
iteration of the "while" loop. Then, the innermost loop starting at line 9 will iterate $t_{j}$ times; inside this loop the complexity is determined by the $l$-shortest path algorithm at line 11 , which takes time $\mathcal{O}\left(l N^{3}\right)$ [14] for a graph with $N$ nodes. Graph $G^{\prime}$ has $n-t_{j}+1$ nodes throughout the innermost loop; the latter then takes time proportional to $l t_{j}\left(n-t_{j}+1\right)^{3}$. For a worst case analysis, we let $t_{j}$, for all iterations $j$, take the value that maximizes the quantity $t_{j}\left(x-t_{j}\right)^{3}$, where $x=n+1$. It is straightforward to show that for this value of $t_{j}$ the complexity of the innermost loop becomes $\mathcal{O}\left(\ln ^{4}\right)$. After accounting for the "while" and outer loops, we conclude that the overall complexity of the algorithm is, in the worst case, $\mathcal{O}\left(k \operatorname{lm} n^{4}\right)$.

Regarding parameters $k$ and $l$, note that the maximum value they can take is, in the worst case, equal to the maximum number of paths of delay at most $\Delta$ between any two nodes in the network. If $\Delta$ is not very loose, we expect the maximum value of both $k$ and $l$ to be a small constant. The actual values of $k$ and $l$ were left unspecified in the description of the algorithm, as in any particular implementation they will be determined by the desired compromise between the quality of the final solution of the algorithm and its speed.

\subsection{Dynamic Reorganization of the Multicast Tree}

In the previous section we presented $D V M A$, an algorithm that can be used during connection establishment to construct a feasible tree for a given destination set. For certain applications, however, it is conceivable that nodes join or leave the initial multicast group during the lifetime of the multicast connection ${ }^{2}$. More specifically, we assume that nodes currently in the multicast group may leave the group after issuing a leave request. Similarly, nodes that wish to join an ongoing multicast session must first issue a join request. Under such a scenario, it is necessary to dynamically update the multicast tree in response to changes in multicast group membership, in order to ensure that constraints (1) and (2) are always satisfied for the current destination set.

Let $T$ be the multicast tree of an ongoing multicast session with destination set $M$, and suppose that as a result of a join or leave request the new destination set is $M^{\prime}$. One possible way of approaching this dynamic version of the DVBMT problem ${ }^{3}$ would be to run DVMA anew to obtain a feasible tree $T^{\prime}$ for set $M^{\prime}$, and, following a transition period, use the new tree for routing subsequent packets of this session. Note that there is a certain overhead associated with this approach, including the computational cost of running $D V M A$, and the cost of the network resources

\footnotetext{
${ }^{2}$ For instance, teleconferencing is an application where the ability to dynamically add or drop parties is highly desirable.

${ }^{3}$ As opposed to the static version we have considered so far, whereby all the destinations in the multicast group are known in advance.
} 
involved in the transition from $T$ to $T^{\prime}$ (i.e., the cost of tearing down old paths and establishing new ones). Since the new tree $T^{\prime}$ can be significantly different than $T$, this overhead can be very high. Furthermore, such a radical approach may cause receivers totally unrelated to the destination nodes added or deleted to experience disruption in service. All these drawbacks make the strategy just described inappropriate for real-time environments and applications where frequent changes in the destination set are anticipated.

We now adopt a different strategy, one that attempts to minimize both the cost incurred during the transition period, and the disruption caused to the receivers. More specifically, the multicast tree is never modified unless it is absolutely necessary to do so. Even then, the new tree is not computed from scratch, rather, a feasible tree for the new multicast group is constructed by making incremental and localized changes to the old tree. We now describe in detail how the join and leave requests are handled under our approach.

Let us first consider leave requests, and assume that node $v \in M$ decides to end its participation in the multicast session. If $v$ is not a leaf node in the current multicast tree $T$ no action needs to be taken. The new tree $T^{\prime}$ can be the same as $T$, with the only difference being that node $v$ will stop forwarding the multicast packets to its local user. If, however, $v$ is a leaf node of $T$, and in order to avoid wasting bandwidth, tree $T$ has to be pruned to exclude $v$ and, possibly, relay nodes and links used in $T$ solely for forwarding packets to $v$. The new tree $T^{\prime}$ is essentially the same as $T$ except in parts of the path from the source to $v$. We conclude that leave requests are easy to handle, and no destination node (other than $v$ ) needs to notice any difference in terms of the multicast session.

Let us now turn our attention to the actions taken whenever a node $u \notin M$ announces its intention to join the multicast group. We distinguish three cases, as follows.

- $u \notin V_{T}$, i.e., the new node is not part of the multicast tree $T$. Our approach is to augment $T$ to include a path from a node $V \in V_{T}$ to the new node $u$. This can be easily accomplished by letting $T_{i}=T$ and $U=\{u\}$ at lines 5 and 6 , respectively, of $D V M A$ (see Figure 2) and executing the code between lines 7 and 17 to search for a path that would result in a feasible tree for the set $M \cup\{u\}$. Hence, the transition phase involves only the establishment of a new path and does not affect any of the paths from the source to nodes already in the multicast group, allowing the connection to proceed smoothly and without any disruption ${ }^{4}$.

\footnotetext{
${ }^{4}$ If executing this piece of code fails to discover such a path, there are two possible courses of action: (a) run $D V M A$ from scratch for the new multicast group, or (b) deny node $u$ its participation in the multicast session; which course of action to be taken may depend on several factors, such as the nature of the application, the cost of rerouting the connection, etc.
} 
- $u \in V_{T}$, i.e., $u$ is a relay node of $T$, and the path from the source node $s$ to $u$ is such that the delay variation constraint (2) is satisfied for the new multicast group $M^{\prime}=M \cup\{u\}^{5}$. Tree $T$ is then a feasible tree for the new set $M^{\prime}$, and can be used without any change other than having node $u$ now forward multicast packets to its user, in addition to forwarding them to the downstream nodes.

- $u \in V_{T}$, but the path from $s$ to $u$ is such that the delay variation constraint (2) is not satisfied for the new set $M \cup\{u\}$. Consequently, a longer path from $s$ to $v$ has to be found. Let $W \subseteq M$ be the destination nodes in $M$ that are downstream of $u$ (i.e., those destination nodes in the subtree of $T$ rooted at $u$ ). Finding a new path from $s$ to $u$ will definitely affect the paths to these nodes, however, the paths to nodes in $M-W$ need not be affected. Let $T_{1}$ be the tree $T$ after excluding its subtree rooted at $u$. Our approach then is to let $T_{i}=T_{1}$ and $U=W \cup\{u\}$ at lines 5 and 6 , respectively, of $D V M A$ in Figure 2. We then execute the code between lines 7 and 17 to connect the destination nodes in $U$ into tree $T_{1}$. As a result, packets will be routed from $s$ to the nodes in $W$ over new paths in the final tree $T^{\prime}$, but none of the paths to nodes in $M-W$ will change.

As a final observation, besides being minimally disruptive, this approach has the additional advantage that the algorithm used during set-up time to construct an initial tree for the multicast connection, can also be used to reorganize the tree during the lifetime of the session.

\section{$5 \quad$ Numerical Results}

We now consider five different algorithms that can be used to construct multicast trees for a given source and destination set, and compare their performance in terms of the maximum delay variation $\delta_{T}$ among the source-destination paths in the final tree $T$, as defined in (3). The five algorithms studied are:

1. DVMA, the algorithm described in Figure 2. We run this algorithm with $\Delta=0.05 \mathrm{~s}$ and $\delta=0$. This value of $\delta$ was used in order to force the algorithm to go through all possible iterations of the outer for loop and return the tree with the smallest value of $\delta_{T}$ it can find. This last value represents the tightest delay variation tolerance for which a tree can be found using DVMA.

\footnotetext{
${ }^{5}$ Note that the path from $s$ to $u$ will necessarily satisfy the delay constraint (1), as $u$ cannot be a leaf node of $T$.
} 
2. DVMA2, an algorithm very similar to DVMA; it differs from the latter in the way the graph $G^{\prime}$ is constructed at line 10 of Figure 2. More specifically, in addition to excluding all nodes in $V_{i}-\{v\}$ and all links in $A_{i}$, all the nodes in $U-\{u\}$ and their adjacent links are also excluded from the initial graph $G$. The values of parameters $\Delta$ and $\delta$ used are the same as for $D V M A$ above.

3. Dijkstra's algorithm [13] which constructs the shortest paths from the source to any node in the network. The resulting tree is pruned to exclude paths that do not terminate at a destination node, and will be referred to as shortest path tree (SPT).

4. Prim's algorithm [15] which constructs a tree of minimum weight spanning all nodes in the network; in our case, the weight of each link is the delay incurred along the link. This minimum spanning tree (MST) is also pruned, as discussed above.

5. The tradeoff algorithm between the minimum spanning tree heuristic ${ }^{6}$ for the Steiner tree problem [7] and SPT, as presented in [5]. The algorithm operates as follows. First a tree is constructed using the heuristic ${ }^{7}$; then the destinations with the largest difference between the delay of their shortest path and the delay of their path in this tree are found, and the tree paths are replaced by the shortest paths from the source to those destinations. This algorithm (which we will call $T D F$ ) is studied because it was conjectured in [10] that it may yield good results in terms of maximum inter-destination delay variation.

We are interested in studying the average case behavior of the five algorithms. To this end, we have generated random graphs for a wide range of values for the total number $n$ of nodes, the average degree of each node, and the number $m$ of destinations in the multicast group as a percentage of $n$. These graphs were constructed to resemble real-world networks using the method described in [4]; the nodes of the graphs were placed in a grid of dimensions $4900 \times 4900 \mathrm{Km}^{8}$ (roughly the size of the continental United States), and the delay along each link was set to the propagation delay of light along that link ${ }^{9}$. The random networks were then fed as input to each algorithm, and the maximum inter-destination delay variation $\delta_{T}$ of the final tree was computed as in expression (3). All figures in this section plot $\delta_{T}$ against the number of nodes $n$ in the

\footnotetext{
${ }^{6}$ Not to be confused with Prim's algorithm for constructing an MST.

${ }^{7}$ The delay along each link is used as the cost of the link.

${ }^{8}$ Hence, the value of $\Delta=0.05 \mathrm{~s}$ corresponds to the time it would take light to travel, at a speed of $2 \times 10^{8} \mathrm{~m} / \mathrm{s}$, over a distance approximately equal to 1.5 times the diameter of this square.

${ }^{9}$ Note that, unless the links operate at or close to their capacity, propagation delays are expected to dominate over queueing and transmission delays in a high-speed environment.
} 
network, for the five algorithms discussed above. Each point plotted represents the average over three hundred different graphs for the stated values of $n, m$, and the average degree of each node.

We first consider networks with average nodal degree equal to 2.5. The results shown in the four Figures 3 - 6 correspond to multicast groups of sizes equal to $5 \%, 10 \%, 15 \%$, and $20 \%$ of the total number of nodes in the network, respectively ${ }^{10}$. From the figures, we can make the following observations regarding the relative performance of the five algorithms. The trees constructed by $D V M A$ and $D V M A 2$ have a maximum delay variation that is always smaller than that of the SPT, $T D F$, and MST trees. Furthermore, DVMA2 outperforms DVMA in most cases shown. A plausible explanation for this fact would be that the small change in the way graph $G^{\prime}$ is constructed at line 10 of the algorithms allows DVMAD to explore a larger number of candidate paths and discover a better overall solution. On the other hand, the $M S T$ is by far the worst tree in terms of $\delta_{T}$; this is expected as Prim's algorithm minimizes the total weight of the tree, without paying any attention to the individual source-destination paths. The tree of shortest paths SPT results in values of $\delta_{T}$ that are between those of the MST and those of the DVMA and DVMA2 algorithms. Note that, in this tree, the value of $\delta_{T}$ is determined by the difference between the delays along the paths to the destinations that are closest and farthest away from the source. Finally, the tradeoff algorithm $T D F$ constructs trees with maximum delay variation larger than that of $S P T$, a result that is in contrast to the expectations expressed in [10].

Let us now turn our attention to how the size $m$ of the multicast group relative to the size $n$ of the network affects the performance of the algorithms. From Figure 3 where $m$ is a small percentage (5\%) of $n$, we can see that the DVMA and DVMA2 trees represent an improvement of roughly an order of magnitude over the SPT and TDF trees. As $m$ increases as a percentage of $n$, the magnitude of improvement decreases, as seen in Figures 4, 5, and 6 which show results for $m$ equal to $10 \%, 15 \%$, and $20 \%$ of $n$, respectively (however, even in Figure 6 with $m=0.2 n$, DVMA2 constructs trees which, on the average, have a value of $\delta_{T}$ at least $16 \%$ lower than that of $\left.S P T\right)$. This behavior can be explained by noting that the smaller the size of the multicast group, the easier it is for DVMA and DVMA2 to find alternative (i.e., longer) paths for the nodes physically closer to the source. At the other extreme, when $m=n$ (a broadcast scenario) it is easy to see that no tree can do much better than SPT in terms of the maximum delay variation. In fact, as the trend in Figures 3 - 6 suggests, when the size of the multicast group is larger than 25-30\% of $n$, it makes sense to simply use the SPT, as running DVMA or DVMA2 would not yield a significant improvement in terms of $\delta_{T}$. In a typical multicast scenario however, the size of the destination

\footnotetext{
${ }^{10}$ There is no point plotted in Figure 3 for $n=20$ as in this case a multicast group of size $0.05 n$ contains only one node, and the quantity $\delta_{T}$ is not meaningful.
} 
set for any single session would be small compared to the total number of nodes (especially for large networks); it is in these situations that the algorithms presented here would really make a difference in terms of the maximum delay variation of the final tree.

Finally, Figures 7 and 8 investigate how the value of $\delta_{T}$ for the various trees changes as a function of the average nodal degree. By comparing these figures to Figure 3 which presents plots for the same size of destination set $(m=0.05 n)$, we see that for the $D V M A$ and $D V M A 2$ trees $\delta_{T}$ decreases dramatically as the average nodal degree increases from 1.5 (Figure 7) to 2.5 (Figure 3) and then to 4 (Figure 8). This is a result of the fact that a higher nodal degree translates into a larger number of paths between any two nodes, and a larger number of trees for our algorithms to choose from. Another important observation is that for an average nodal degree equal to 4 , both of our algorithms are able to construct trees with $\delta \approx 0$, independently of the number of nodes in the network. As such, these trees would be able to meet the delay variation requirements of even the most demanding applications. The behavior of the other algorithms is not significantly affected by the nodal degree, as none of these attempt to optimize in terms of $\delta_{T}$. In $S P T$, for instance, $\delta_{T}$ is determined by the relative distance of the various destinations from the source, which is almost independent of the nodal degree.

Overall, the results presented in this section suggest that DVMA and DVMA2 achieve their best performance under conditions that are typical of multicast applications running in high speed networks, namely, when (a) the size of the multicast group is relatively small compared to the total number of nodes in the network, and/or (b) the number of incoming/outgoing links at each node is relatively large.

\section{Concluding Remarks}

We have considered the problem of determining multicast trees that guarantee certain bounds on the end-to-end delays from the source to the each of the destination nodes, as well as on the variation among these delays. The bounds are directly related to the quality of service requirements of the higher level applications performing the multicast. After establishing that the problem of constructing such constrained trees is $\mathcal{N} \mathcal{P}$-complete, we developed heuristics that exhibit good average case behavior. Our heuristics perform especially well under conditions typical of multicast scenarios in high-speed networks, namely, when the network is not too sparse, and when the number of destination nodes is relatively small compared to the total number of nodes. We have also shown that the strategy employed by the heuristic is applicable to the problem of reorganizing the tree in response to changes in multicast group membership. 
Our work can be extended in several directions. Recall that our algorithms do not attempt to optimize the tree in terms of cost; in fact, since their strategy is to choose longer paths for some of the destination nodes, the cost of the final tree may be somewhat high. One straightforward approach to dealing with cost is to modify our algorithms to seek the least cost path among the candidate paths they consider, and/or the least cost tree among all feasible trees they construct. However, this issue warrants further investigation. We also plan to develop new heuristics for multipoint-to-multipoint communication, whereby a set of sources and a set of destination nodes share the same multicast tree. 


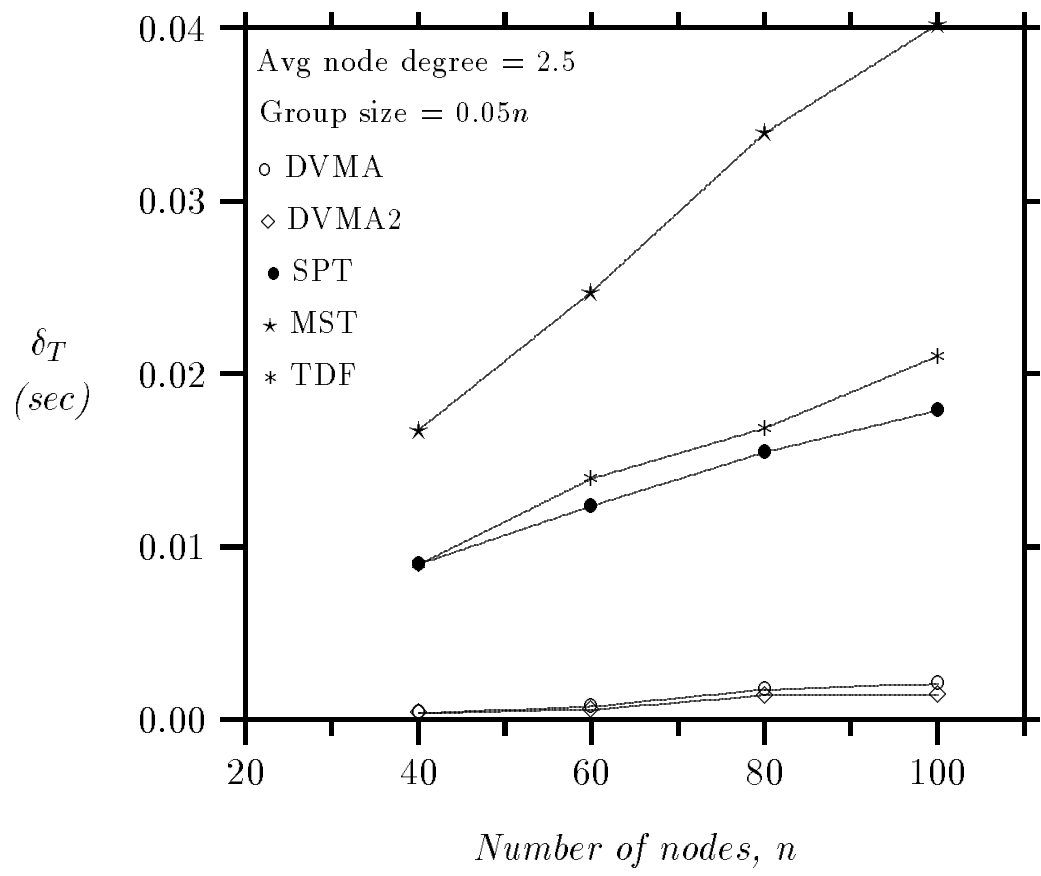

Figure 3: Algorithm comparison for networks with average node degree equal to 2.5, and multicast group size equal to $5 \%$ of the number of nodes

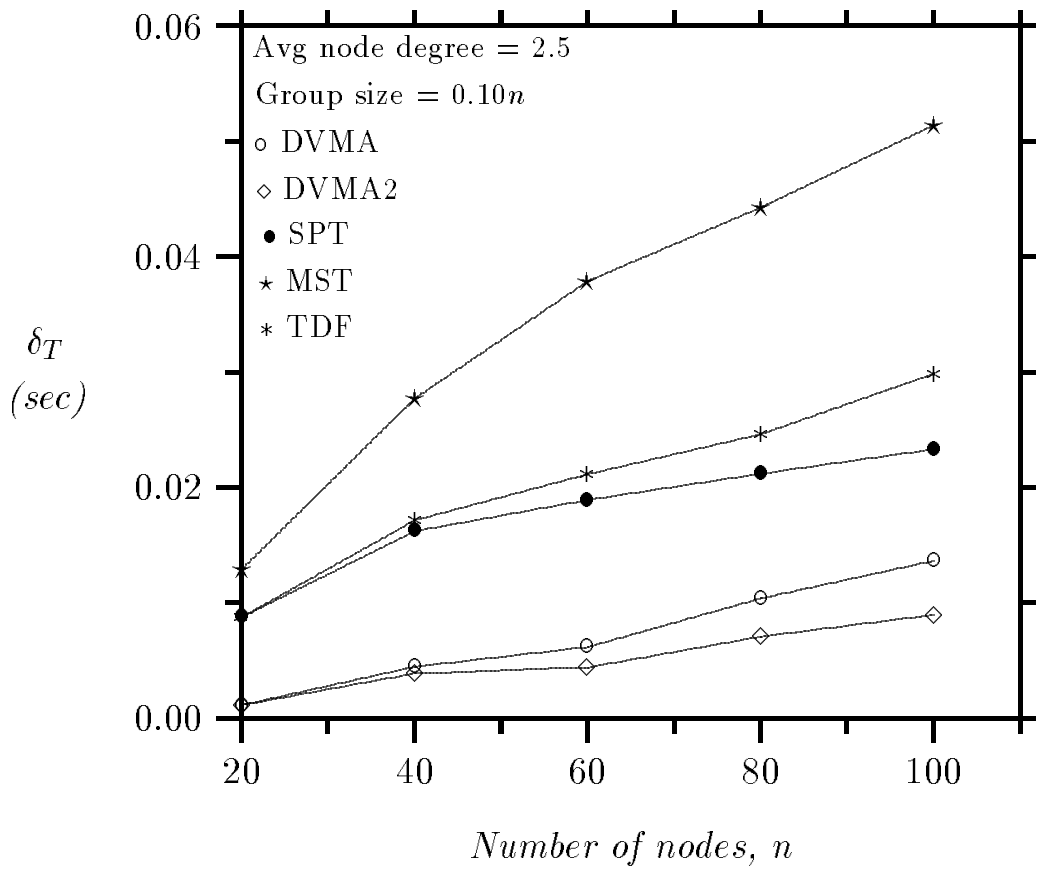

Figure 4: Algorithm comparison for networks with average node degree equal to 2.5, and multicast group size equal to $10 \%$ of the number of nodes 


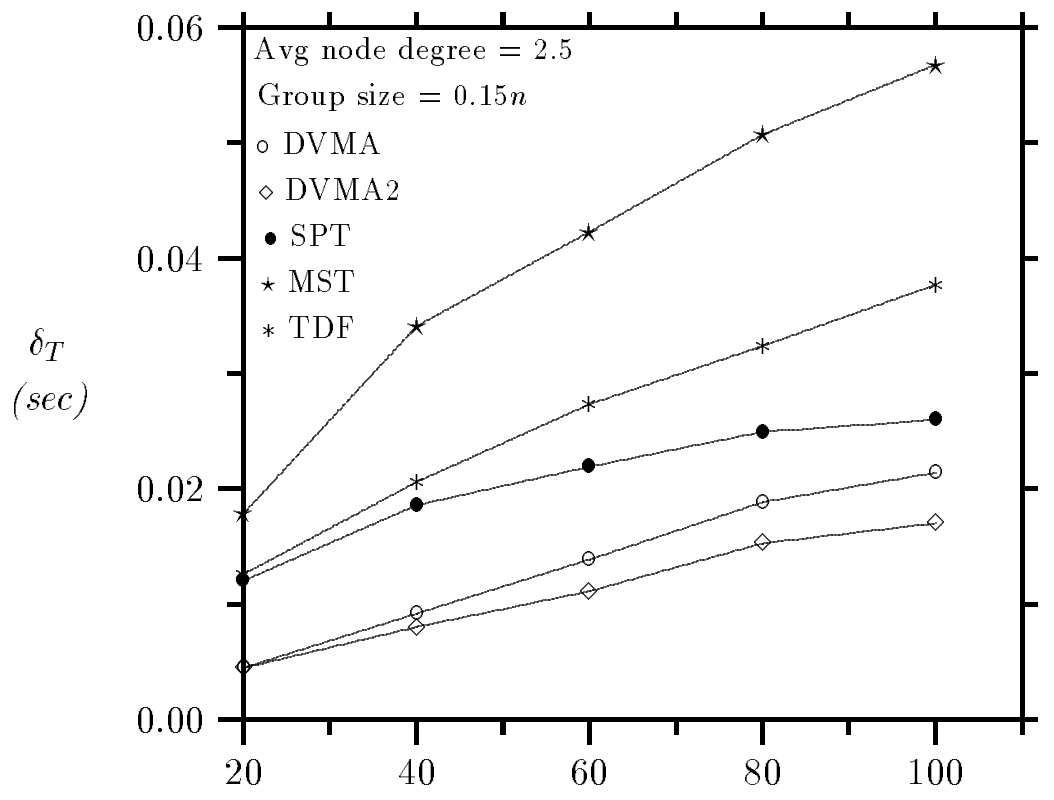

Number of nodes, $n$

Figure 5: Algorithm comparison for networks with average node degree equal to 2.5, and multicast group size equal to $15 \%$ of the number of nodes

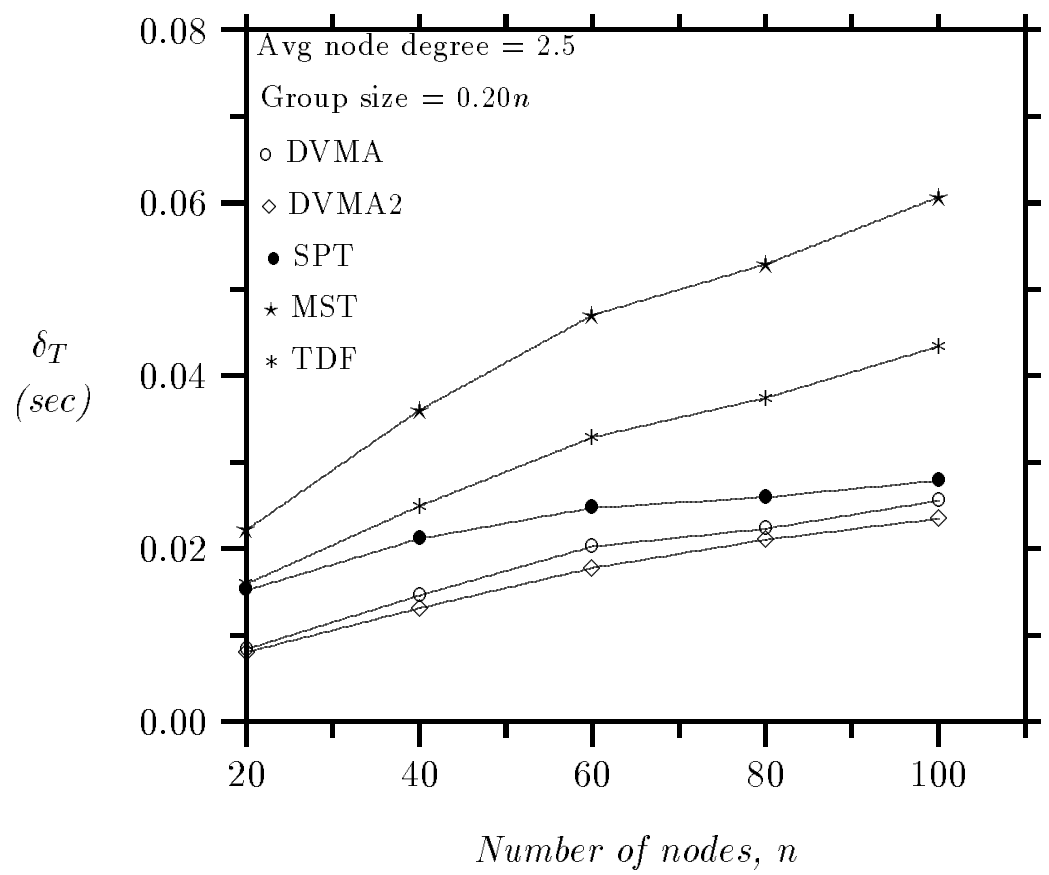

Figure 6: Algorithm comparison for networks with average node degree equal to 2.5, and multicast group size equal to $20 \%$ of the number of nodes 


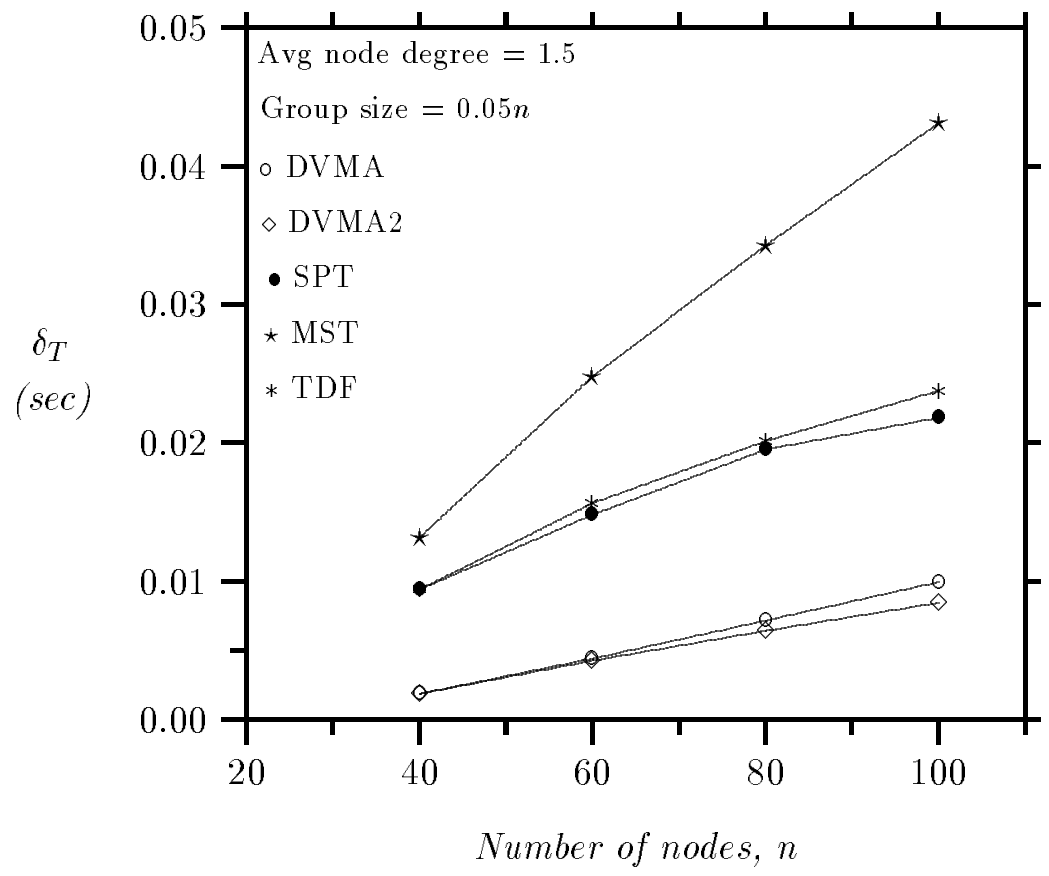

Figure 7: Algorithm comparison for networks with average node degree equal to 1.5, and multicast group size equal to $5 \%$ of the number of nodes

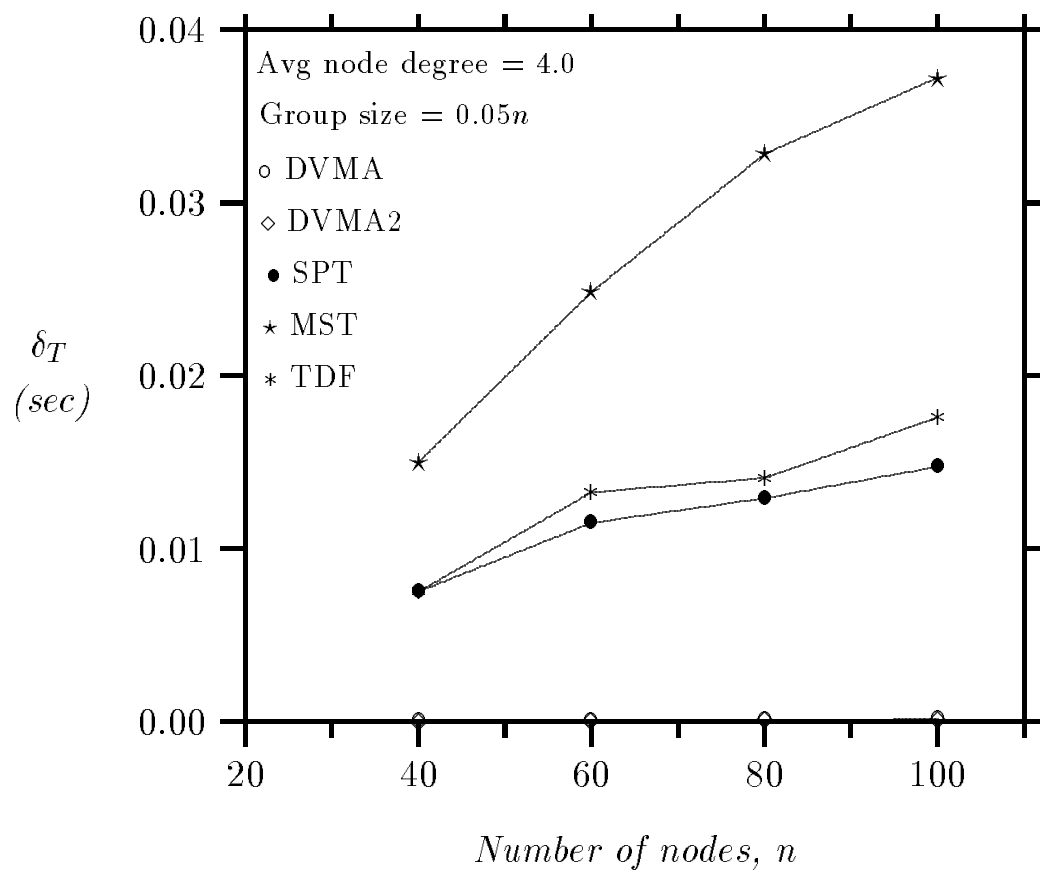

Figure 8: Algorithm comparison for networks with average node degree equal to 4, and multicast group size equal to $5 \%$ of the number of nodes 


\section{References}

[1] J. S. Turner. New directions in communications (or which way to the information age?). IEEE Communications Magazine, 24(10):8-15, October 1986.

[2] S. L. Hakimi. Steiner's problem in graphs and its implications. Networks, 1:113-133, 1971.

[3] M. R. Garey, R. L. Graham, and D. S. Johnson. The complexity of computing steiner minimal trees. SIAM Journal of Applied Mathematics, 32(4):835-859, June 1977.

[4] B. W. Waxman. Routing of multipoint connections. IEEE Journal on Selected Areas in Communications, 6(9):1617-1622, December 1988.

[5] K. Bharath-Kumar and J. M. Jaffe. Routing to multiple destinations in computer networks. IEEE Transactions on Communications, COM-31(3):343-351, March 1983.

[6] L. Kou, G. Markowsky, and L. Berman. A fast algorithm for steiner trees. Acta Informatica, 15:141-145, 1981.

[7] E. N. Gilbert and H. O. Pollak. Steiner minimal tree. SIAM Journal on Applied Mathematics, 16, 1968.

[8] V. P. Kompella, J. C. Pasquale, and G. C. Polyzos. Multicast routing for multimedia communication. IEEE/ACM Transactions on Networking, 1(3):286-292, June 1993.

[9] Q. Zhu, M. Parsa, and J. J. Garcia-Luna-Aceves. A source-based algorithm for near-optimum delay-constrained multicasting. In Proceedings of IEEE Infocom '95, March 1995.

[10] H. Salama, D. Reeves, Y. Viniotis, and T. Sheu. Comparison of multicast routing algorithms for high-speed networks. Technical Report IBM-TR29.1930, IBM, September 1994.

[11] H. Salama, D. Reeves, Y. Viniotis, and T. Sheu. Evaluation of multicast routing algorithms for distributed real-time applications in high-speed networks. In Proc. of 6th IFIP Conf. on High Speed Networks, September 1995.

[12] D. Bertsekas and R. Gallager. Data Networks. Prentice Hall, Inc., 1992.

[13] E. W. Dijkstra. A note on two problems in connexion with graphs. Numerische Mathematik, $1: 269-271,1959$.

[14] E. Lawler. Combinatorial Optimization: Networks and Matroids. Holt, Rinehart and Winston, 1976. 
[15] R. C. Prim. Shortest connection networks and some generalizations. Bell Systems Technical Journal, 36:1389-1401, Nov 1957.

[16] M. R. Garey and D. S. Johnson. Computers and Intractability. W. H. Freeman and Co., New York, 1979. 


\section{A Proof that Problem $D V B M T$ is $\mathcal{N} \mathcal{P}$-complete}

We now show that problem $D V B M T$ is $\mathcal{N} \mathcal{P}$-complete even when the number of destination nodes is $|M|=2$. The proof uses a transformation from PARTITION, a well known $\mathcal{N} \mathcal{P}$-complete problem [16], repeated here for the sake of completeness.

Problem A.1 (PARTITION) Given a set of $k$ elements $S=\{1,2, \ldots, k\}$ with $a_{i}$ the weight of element $i$, and $A=\sum_{i=1}^{k} a_{i}$, does there exist a partition of $S$ into two sets, $S_{1}$ and $S_{2}$, such that $\sum_{i \in S_{1}} a_{i}=\sum_{j \in S_{2}} a_{j}=\frac{A}{2}$ ? (The $a_{i}$ 's may be assumed integer.)

We are now ready to prove Theorem 3.1.

Proof (of Theorem 3.1). It is easy to see that DVBMT is in the class $\mathcal{N P}$, since a nondeterministic algorithm need only guess a tree spanning $s$ and the nodes in the destination set $M$, and verify in polynomial time that the tree is a feasible one (i.e., that it satisfies both (1) and (2)).

We now transform PARTITION to DVBMT; note that it is sufficient to find a transformation for the case $|M|=2$. Let $S=\{1,2, \ldots, k\}$ be the set of elements of weights $a_{i}, i=1, \ldots, k$, making up an arbitrary instance of $P A R T I T I O N$, and let $A=\sum_{i=1}^{k} a_{i}$. We construct an instance of DVBMT as follows (see Figure 9). The network $G=(V, A)$ has $n=k+3$ nodes, with $V=\left\{s, v, u, r_{1}, r_{2}, \ldots, r_{k}\right\}$, where $s$ is the source node and $M=\{v, u\}$ is the destination set. The set $A$ of links is:

$$
\begin{aligned}
A= & \left\{(s, v),\left(s, r_{1}\right), \ldots,\left(s, r_{k}\right),\left(r_{1}, u\right), \ldots,\left(r_{k}, u\right),\right. \\
& \left.\left(r_{1}, r_{2}\right), \ldots,\left(r_{1}, r_{k}\right),\left(r_{2}, r_{1}\right),\left(r_{2}, r_{3}\right), \ldots,\left(r_{2}, r_{k}\right), \ldots,\left(r_{k}, r_{1}\right), \ldots,\left(r_{k}, r_{k-1}\right)\right\}
\end{aligned}
$$

In other words, there is a directed link from $s$ to $v$, one link from $s$ to each node $r_{i}$, one link from each node $r_{i}$ to $u$, and one link from $r_{i}$ to $r_{j}, i, j=1, \ldots, k, i \neq j$ (i.e., the subgraph of $G$ containing only nodes $r_{i}, i=1, \ldots, k$, is a complete graph on these nodes). As we can see, there is only one path from $s$ to destination node $v$ consisting of the single link $(s, v)$; however, a path from $s$ to the other destination $u$ may contain any number of the nodes $r_{i}, i=1, \ldots, k$, and in any order (refer also to Figure 9).

We now define the link-delay function $\mathcal{D}$ for this instance of $D V B M T$ as:

$$
\mathcal{D}(\ell)= \begin{cases}\frac{A}{2}, & \text { if } \ell=(s, v) \\ 0, & \text { if } \ell=(x, u), x \in V \\ a_{i}, & \text { if } \ell=\left(x, r_{i}\right), x \in V\end{cases}
$$

As a result of this definition, if the path from $s$ to $u$ passes through node $r_{i}$ for some $i$, then a delay equal to $a_{i}$ is incurred along the link that leads to $r_{i}$. Finally, the delay and delay variation tolerances are $\Delta=\frac{A}{2}$, and $\delta=0$, respectively. 


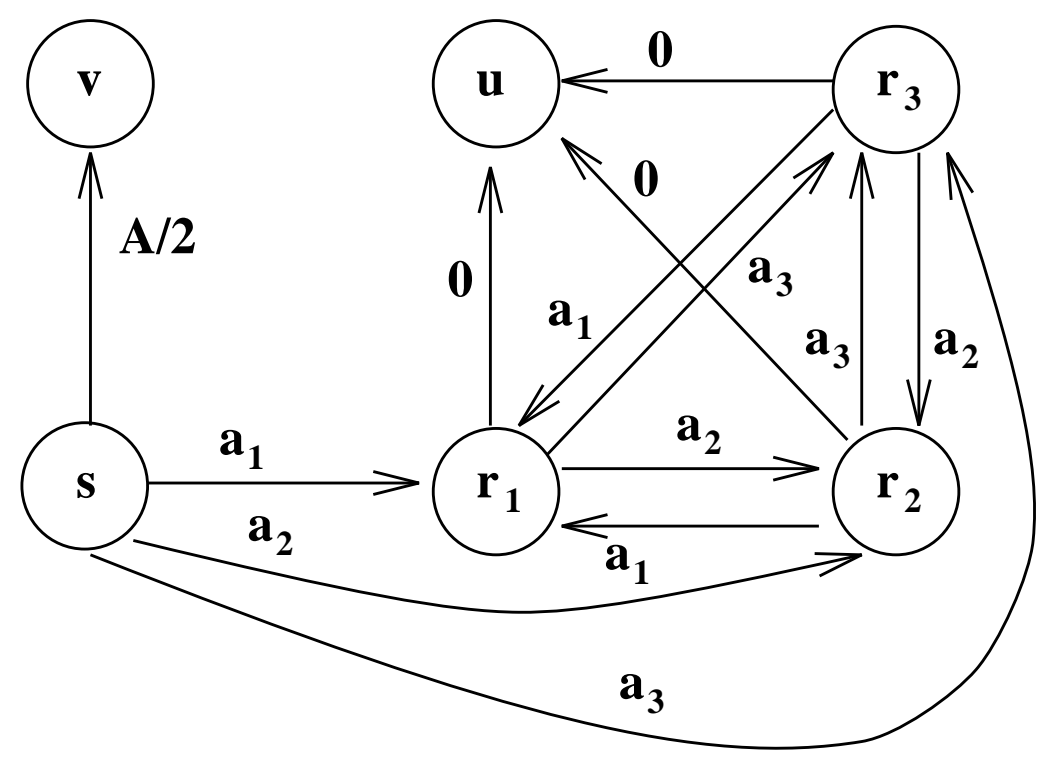

Figure 9: Instance of DVBMT corresponding to an instance of PARTITION with $S=\{1,2,3\}$

It is obvious that this transformation can be performed in polynomial time. We now show that a feasible tree exists for the above instance of $D V B M T$ if and only if set $S$ has a partition. If $S$ has a partition $S_{1}, S_{2}$, then $S_{1}=\left\{a_{\pi_{1}}, \ldots, a_{\pi_{l}}\right\}$ for some $l<k$. The tree consisting of path $(s, v)$ and path $\left(s, r_{\pi_{1}}\right),\left(r_{\pi_{1}}, r_{\pi_{2}}\right), \ldots,\left(r_{\pi_{l-1}}, r_{\pi_{l}}\right),\left(r_{\pi_{l}}, u\right)$, is then a feasible tree for DVBMT, as the delay along both paths is equal to $\frac{A}{2}$.

Conversely, let $T$ be a feasible tree for DVBMT. Then $T$ must include the path $(s, v)$ of delay $\frac{A}{2}$, as this is the only path from the source to $v$. Let $\left(s, r_{\pi_{1}}\right),\left(r_{\pi_{1}}, r_{\pi_{2}}\right), \ldots,\left(r_{\pi_{l-1}}, r_{\pi_{l}}\right),\left(r_{\pi_{l}}, u\right)$, be the path from $s$ to $u$ on tree $T$. Since $T$ is a feasible tree and $\delta=0$, the delay along the latter path is equal to $\frac{A}{2}$, and $l<k$ (for if $l=k$, the path from $s$ to $u$ would include all $r_{i}, i=1, \ldots, k$, and the delay along the path would equal $A$, contradicting our hypothesis that $T$ is a feasible tree). Then, $\sum_{i=1}^{l} a_{\pi_{i}}=\frac{A}{2}$, implying that $S_{1}=\left\{a_{\pi_{1}}, \ldots, a_{\pi_{l}}\right\}, S_{2}=S-S_{1} \neq \phi$, is a partition of $S$. 